\title{
Apparent electron-phonon interaction in strongly correlated systems
}

\author{
O. Rösch and O. Gunnarsson \\ Max-Planck-Institut für Festkörperforschung, D-70506 Stuttgart, Germany
}

\begin{abstract}
We study the interaction of electrons with phonons in strongly correlated solids, having high- $T_{c}$ cuprates in mind. Using sum-rules, we show that the apparent strength of this interaction strongly depends on the property studied. If the solid has a small fraction (doping) $\delta$ of charge carriers, the influence of the interaction on the phonon self-energy is reduced by a factor $\delta$, while there is no corresponding reduction of the coupling seen in the electron self-energy. This supports the interpretation of recent photoemission experiments, assuming a strong coupling to phonons.
\end{abstract}

There has been much interest in electron-phonon coupling in high- $T_{c}$ cuprates after photoemission spectroscopy (PES) studies of Lanzara et al. [1] showed a strong coupling to a mode at $70 \mathrm{meV}$. This was interpreted as a coupling to a half-breathing phonon, where $\mathrm{O}$ atoms in the $\mathrm{CuO}_{2}$ plane move towards $\mathrm{Cu}$ atoms. This interpretation is supported by the anomalous softening under doping [2, 3, 4, 5] and large width [3] of this phonon. It is intriguing, however, that a substantially larger apparent coupling strength $\lambda$ was estimated from a kink in the PES spectrum [1] than what is suggested from the phonon width and softening [6]. Similar considerations apply to the $\mathrm{B}_{1 g}$ phonon width [7] and the anomaly at $40 \mathrm{meV}$ seen in PES [8]. These estimates of $\lambda$ were based on theories which assume noninteracting electrons. Using sum-rules, we show that for a strongly correlated system the apparent $\lambda$ deduced from such theories should depend strongly on the property studied. We consider a doped Mott (charge transfer) insulator, such as a cuprate, which has a fraction $\delta$ carriers (where $\delta$ typically is small). The influence of the electron-phonon interaction on the phonon self-energy, determining its width and softening, is then reduced by a factor of the order of $\delta$ compared to a system without electron-electron interaction. For the electron self-energy, determining the photoemission spectrum, there is no comparable reduction. This explains why the $\lambda$ deduced from the phonon self-energy appears smaller than the one deduced from PES, and it supports the scenario that phonons give a large contribution to structures in PES. For similar reasons, there should be no reduction $\sim \delta$ in the phonon induced carrier-carrier interaction. To address these issues, we present a method for determining $\lambda$ in exact diagonalization approaches.

The electron-electron interaction can strongly reduce the effects of the electron-phonon interaction on phonons [9]. The electron density is rearranged in response to the excitation of a phonon, and this rearrangement acts back on the phonon, contributing to the width and energy shift of the phonon. The response of the electrons depends on electron hopping, which is hindered by interaction effects. In particular, if the interaction is strong enough to lead to a Mott insulator (for $\delta=0$ ), the electron-phonon contribution to the phonon width vanishes.

For cuprates, this can be studied in the $t-J$ model [10], which has one site per $\mathrm{Cu}$ atom. Each site is occupied by either a $\mathrm{Cu} 3 d$-hole or a Zhang-Rice singlet, composed of a $\mathrm{Cu} 3 d$-hole and an $\mathrm{O} 2 p$-hole. The doping $\delta$ gives the fraction of singlets, which provide the charge carriers. The Hamiltonian is given by

$$
\begin{aligned}
H_{t-J} & =J \sum_{<i, j>}\left(\mathbf{S}_{i} \cdot \mathbf{S}_{j}-\frac{n_{i} n_{j}}{4}\right) \\
& -t \sum_{<i, j>\sigma}\left(\tilde{c}_{i \sigma}^{\dagger} \tilde{c}_{j \sigma}+H . c .\right)
\end{aligned}
$$

where $\tilde{c}_{i \sigma}^{\dagger}$ creates a $d$-hole on site $i$ if this site previously had no hole. A hole can hop with the hopping integral $t$ to sites occupied by singlets and vice versa. The spins of the $3 d$-holes have a Heisenberg interaction with the interaction strength $J$. In the $t-J$ model with phonons 11, 12, 13, 14, 15, 16], the phonons couple mainly to the on-site energies and only weakly to the terms describing hopping and spin-spin interaction [11, 15. In the following we only include the coupling to the on-site term,

$$
H_{e p}=\frac{1}{\sqrt{N}} \sum_{i, \mathbf{q}} g_{\mathbf{q}}\left(n_{i}-1\right)\left(b_{\mathbf{q}}+b_{-\mathbf{q}}^{\dagger}\right) e^{i \mathbf{q} \cdot \mathbf{R}_{i}},
$$

where $N$ is the number of sites, $g_{\mathbf{q}}$ is a coupling constant, $n_{i}$ measures the $d$-hole occupation on the site at $\mathbf{R}_{i}$ and $b_{\mathbf{q}}$ annihilates a phonon with a wave vector $\mathbf{q}$.

The phonons only couple to sites with Zhang-Rice singlets, i.e., sites with no $d$-holes. The phonon self-energy $\Pi(\mathbf{q}, \omega)$ can then be expressed in terms of the chargecharge response function $\chi(\mathbf{q}, \omega)$.

$$
\Pi(\mathbf{q}, \omega)=\frac{\left(g_{\mathbf{q}}^{2} / N\right) \chi(\mathbf{q}, \omega)}{1+\left(g_{\mathbf{q}}^{2} / N\right) \chi(\mathbf{q}, \omega) D_{0}(\mathbf{q}, \omega)},
$$

where $D_{0}(\mathbf{q}, \omega)$ is the free phonon Green's function. Khaliullin and Horsch 12] showed that there is a sumrule

$$
\frac{1}{\pi N} \sum_{\mathbf{q} \neq 0} \int_{-\infty}^{\infty}|\operatorname{Im} \chi(\mathbf{q}, \omega)| d \omega=2 \delta(1-\delta) N,
$$

This result is a factor $2 \delta(1-\delta)$ times the result for noninteracting electrons in a half-filled band. Since $\chi(\mathbf{q}, \omega)$ becomes small for small $\delta$, the same is true for $\Pi(\mathbf{q}, \omega)$. 
In this limit the denominator in Eq. (3) is not very important, and the sum-rule in Eq. (4) also applies approximately to $\Pi(\mathbf{q}, \omega) / g_{\mathbf{q}}^{2}$.

To understand the result in Eq. (4), we notice that the system can respond to the perturbation of a phonon by transferring singlets to sites with $\mathrm{Cu} 3 d$-holes. If there are few singlets, i.e., $\delta$ is small, the response of the system is weak and the phonon self-energy is small. Since typically $\delta \sim 0.1$, this drastically reduces the phonon softening and width.

It is interesting to study PES and inverse PES (IPES) to see if many-body effects also drastically change the effects of the electron-phonon interaction in these cases. These spectra are described by the spectral function $A(\mathbf{k}, \omega)=\operatorname{Im} G\left(\mathbf{k}, \omega-i 0^{+}\right) / \pi$, where $\mathbf{k}$ is a momentum and $\omega$ a frequency. $G(\mathbf{k}, z)$ is the electron Green's function

$$
G(\mathbf{k}, z)=\frac{a_{\mathbf{k}}}{z-\varepsilon_{\mathbf{k}}-\Sigma(\mathbf{k}, z)},
$$

where $a_{\mathbf{k}}$ is a weight, $z$ is a complex number and $\Sigma(\mathbf{k}, z)$ is the electron self-energy. The $z$-independent part of $\Sigma$ is included in the energy $\varepsilon_{\mathbf{k}}$, so that $\Sigma(\mathbf{k}, z) \sim b_{\mathbf{k}} / z$ for large $z$. To determine $b_{\mathbf{k}}$, we expand the Green's function in $1 / z$

$$
\begin{aligned}
G(\mathbf{k}, z) & =\frac{a_{\mathbf{k}}}{z}\left(1+\frac{\varepsilon_{\mathbf{k}}}{z}+\frac{\varepsilon_{\mathbf{k}}^{2}+b_{\mathbf{k}}}{z^{2}}+. .\right) \\
& =\frac{a_{\mathbf{k}}}{z}\left(1+\frac{\langle\omega\rangle_{\mathbf{k}}}{z}+\frac{\left\langle\omega^{2}\right\rangle_{\mathbf{k}}}{z^{2}}+. .\right),
\end{aligned}
$$

where $\left\langle\omega^{n}\right\rangle_{\mathbf{k}}=\int \omega^{n} A(\mathbf{k}, \omega) d \omega / \int A(\mathbf{k}, \omega) d \omega$. The coefficient $b_{\mathbf{k}}$ can then be expressed in terms of $\langle\omega\rangle_{\mathbf{k}}$ and $\left\langle\omega^{2}\right\rangle_{\mathbf{k}}$. Using the analytical properties, we can relate $b_{\mathbf{k}}$ to a sum-rule over $\operatorname{Im} \Sigma\left(\mathbf{k}, \omega-i 0^{+}\right)$. Since there are no phonons in the ground-state in the model (2) for $\delta=0$, we can easily calculate expectation values of powers of $H_{e p}$, needed to obtain $\left\langle\omega^{n}\right\rangle_{\mathbf{k}}$. From this we deduce a sum-rule for the difference $\Sigma_{e p}$ between the self-energies with and without electron-phonon interaction.

$$
\frac{1}{\pi} \int_{-\infty}^{0} \operatorname{Im} \Sigma_{e p}\left(\mathbf{k}, \omega-i 0^{+}\right) d \omega=\frac{1}{N} \sum_{\mathbf{q}}\left|g_{\mathbf{q}}\right|^{2} \equiv \bar{g}^{2},
$$

which is valid for $\delta=0$. The integration only runs over energies corresponding to the PES spectrum, since the weight of the IPES spectrum is zero for $\delta=0$. The result (77) is equal to the lowest order result (in $g_{\mathbf{q}}^{2}$ ) for noninteracting electrons. While the effect of the electronphonon coupling is strongly reduced by the small doping for the phonon self-energy, there is no such reduction for the electron self-energy. $\operatorname{Re} \Sigma_{e p}$ is related to $\operatorname{Im} \Sigma_{e p}$ via a Hilbert transform.

The sum-rule in Eq. (7) can be understood if we notice that a singlet with the wave vector $\mathbf{k}$, created in PES, can easily be scattered by phonons to other states $\mathbf{k}+\mathbf{q}$, since only a fraction $(1-\delta)$ of these are occupied by other singlets. We therefore expect a strong electron-phonon interaction in PES also for a finite but small $\delta$. As a result, one would then expect that the electron-phonon interaction appears to be a factor of $1 /(c \delta)$ stronger in $\operatorname{Re} \Sigma(\mathbf{q}, \omega)$ than for the phonon width $2 \operatorname{Im} \Pi(\mathbf{q}, \omega)$, where $c \sim 2-4$ depends on the assumptions about the $\omega$-dependencies of $\operatorname{Im} \Sigma$ and Im $\Pi$. Although the arguments above show that the right hand side of the sumrule in Eq. (7) should not go to 0 for $\delta \rightarrow 0$, the result is, nevertheless, highly nontrivial. The right hand side is independent of $\mathbf{k}, t$ and $J$. It is also interesting that it remains proportional to $\bar{g}^{2}$ for large $\bar{g}$.

From these arguments it follows that the phonon induced attractive carrier-carrier interaction should also be effective, since for small $\delta$ the carriers (singlets) can scatter each other via phonons with few restrictions. This may be helpful for superconductivity. In particular, it should be possible to have a strong phonon induced carrier-carrier interaction, without the corresponding phonon going soft.

To illustrate these points, we study the $t$ - $J$ model with phonons, using exact diagonalization. We consider a finite cluster with $4 \times 3$ sites, and include the entire breathing phonon branch. The Hilbert space is limited by only allowing for states which have a maximum of $K$ phonons, where typically $K=7$. Ideally, we would calculate the PES spectrum as a function of $\mathbf{k}$. We would then expect to see a kink in the dispersion at energies of the order of $\omega_{p h}$ away from the Fermi energy, where $\omega_{p h}$ is a typical phonon energy. For the small clusters which can be studied in exact diagonalization, this approach is not possible, due to the few $\mathbf{k}$-points available.

Instead we focus directly on the self-energy. The spectral function $A(\mathbf{k}, \omega)$ is calculated using exact diagonalization. The Green's function is then obtained from a Kramers-Kronig transformation. Finally, $\Sigma(\mathbf{k}, \omega)$ is obtained by inverting Eq. (5). In a similar way we determine $\Pi(\mathbf{q}, \omega)$ from the phonon spectral function. The $\omega$ dependence of $\operatorname{Re} \Sigma(\mathbf{q}, \omega)$ then gives information about the kink in PES and $\operatorname{Im} \Pi\left(\mathbf{q}, \omega_{p h}\right)$ gives the phonon width.

Due to the small size of the cluster there are few manyelectron states in the phonon energy range, which makes it hard to extract phonon widths, even in the approach above. The main points of the paper can, however, also be illustrated by using a larger phonon energy. We have therefore increased the bare phonon energy to $\omega_{p h}^{0}=0.5$ $\mathrm{eV}$. This requires a corresponding increase in the coupling constant. We have chosen a multiplying factor of 3.4, which leads to an apparent coupling strength to the phonons of the order seen experimentally [17]. We have furthermore used the parameters $t=0.47 \mathrm{eV}$ and $J / t=0.3$.

Figure \shows the frequency integrals of the imaginary parts of the electron and phonon self-energies as a func- 


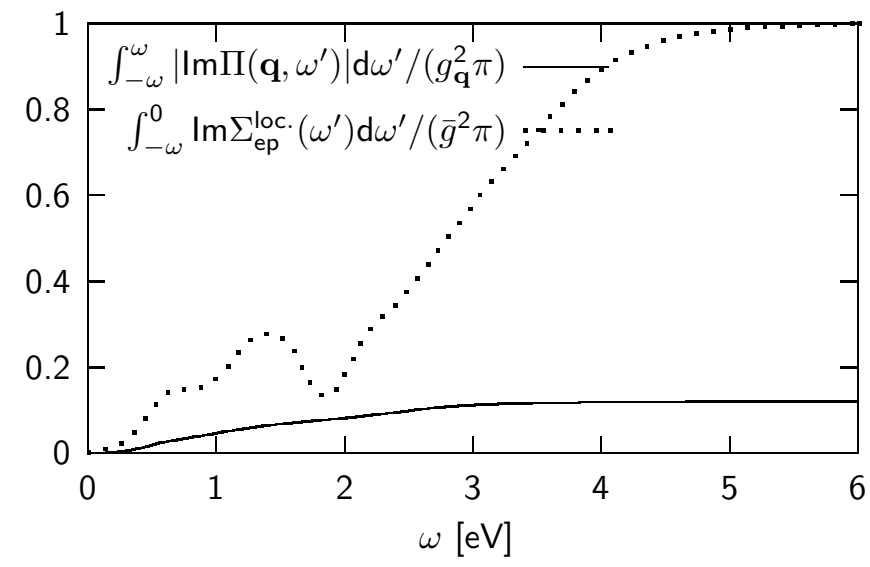

FIG. 1: Frequency integrals over the imaginary parts of the phonon self-energy for $\mathbf{q}=\pi /(2 a)(1,0)$ and the electronphonon contribution to the $\mathbf{k}$-averaged electron self-energy. The results are divided by the coupling constants and they were obtained for a $4 \times 3$ cluster with periodic boundary conditions. $\Pi$ was calculated for $\delta=1 / 12$ and $\Sigma_{e p}$ for $\delta=0$. The self-energies were given a $0.2 \mathrm{eV}$ (FWHM) Lorentzian broadening. The figure illustrates how these quantities converge to approximately $2 \delta \approx 0.17$ [Eq. (4)] and unity [Eq. (7)] for the phonon and electron self-energy, respectively.

tion of the upper limit $\omega$. The limit $\omega \rightarrow \infty$ corresponds to the sum-rules (4) and (7). To obtain dimensionless quantities, the coupling constants have been divided out. The figure illustrates the large ratio, $\sim 1 /(2 \delta)$, of the sum-rules. The sum-rule in Eq. (4) applies to an average over $\mathbf{q} \neq 0$ of $\chi$, but we found that it is also rather accurate for $\Pi / g_{\mathbf{q}}^{2}$ for an individual value of $\mathbf{q}$, as is illustrated in Fig. [1 The reason is that the denominator in Eq. (3) is not very important for the coupling strengths used here and that the sum-rule for an individual $\mathbf{q}$ is not very different from the average over $\mathbf{q}$.

The coupling strength $\lambda$ is often determined from the phonon width $\gamma=2 \operatorname{Im} \Pi\left(\mathbf{q}, \omega_{p h}\right)[18]$ or its softening, $\Delta \omega_{p h}=\operatorname{Re} \Pi\left(\mathbf{q}, \omega_{p h}\right)$, using

$$
\lambda^{\Pi}=\frac{\gamma}{2 \pi \omega_{p h}^{2} N(0)}=-\alpha \frac{\Delta \omega_{p h}}{\omega_{p h}^{0}},
$$

appropriate for noninteracting electrons. Here $N(0)$ is the electron density of states per spin and $\alpha \sim 1$ depends on the precise $\omega$ dependence of $\operatorname{Im} \Pi(\mathbf{q}, \omega)$. Figure 2 shows the broadened $\operatorname{Im} \Pi(\mathbf{q}, \omega)$. The broadening $(0.4 \mathrm{eV}$ FWHM) was chosen in such a way that the fine structures due to the finite cluster size were removed and so that the expected behavior $\operatorname{Im} \Pi(\mathbf{q}, \omega) \sim \omega$ for small $\omega$ was obtained. The phonon is softened to $\omega_{p h}=0.4 \mathrm{eV}$, due to the electron-phonon interaction. For this frequency we obtain the FWHM of the phonon as $\gamma=0.08 \mathrm{eV}$. This result depends on the broadening of $\operatorname{Im} \Pi(\mathbf{q}, \omega)$ and results differing by $\pm 30 \%$ could be obtained for other reasonable broadenings. Based on the width of $\operatorname{Im} \Pi(\mathbf{q}, \omega)$, we estimate $N(0) \sim 0.5$ states per $\mathrm{eV}$ and spin and $\lambda^{\Pi}=0.2$.

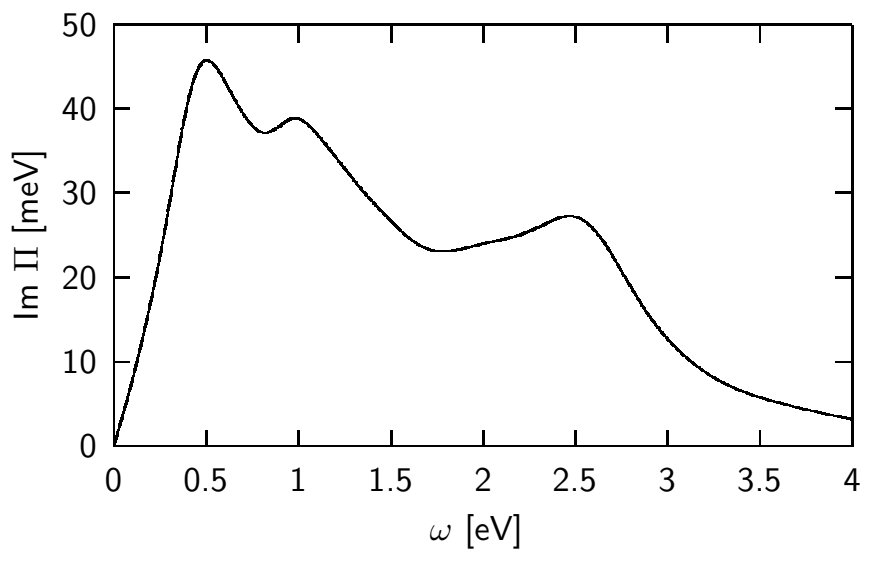

FIG. 2: $\operatorname{Im} \Pi(\mathbf{q}, \omega)$ of a $4 \times 3$ cluster for $\mathbf{q}=\pi /(2 a)(1,0)$ and $\delta=1 / 12$. The self-energy has been given a Lorentzian broadening of $0.4 \mathrm{eV}$.

From the phonon softening $\Delta \omega_{p h} / \omega_{p h}^{0}=0.2$, we obtain a similar result for $\lambda^{\Pi}$.

We emphasize the difference in this approach, using a broadened $\Pi$, from an approach where the phonon spectral function is broadened until a smooth spectrum is obtained. The latter approach leads to an additional width of the peaks. For the present system, such a large broadening would be required that the approach would not be meaningful. The broadening of $\operatorname{Im} \Pi(\mathbf{q}, \omega)$, on the other hand, does not generally add to the width of the phonon spectral function, since it essentially only distributes the contributions to $\operatorname{Im} \Pi(\mathbf{q}, \omega)$ more uniformly in $\omega$.

We next consider the coupling strength seen in the electron self-energy determining PES. We can determine a $\lambda^{\Sigma}$ as

$$
\lambda^{\Sigma}=-\left.\frac{d \operatorname{Re} \Sigma_{e p}(\omega)}{d \omega}\right|_{\omega=0}=\frac{1}{\pi} \int d \omega \frac{\operatorname{Im} \Sigma_{e p}(\omega)}{\omega^{2}} .
$$

This leads to $\lambda^{\Sigma}=0.6$, which is about a factor of $1 /(c \delta)$ larger than $\lambda^{\Pi}$ with $c=4$. As can be seen from Fig. [1 $\operatorname{Im} \Sigma$ is small for $|\omega|<\omega_{p h}=0.4 \mathrm{eV}$. For this frequency range we find that $\operatorname{Re} \Sigma(\mathbf{k}, \omega) \sim-\lambda^{\Sigma} \omega$.

To summarize, we have found that in strongly correlated systems the apparent strength $\lambda$ of the electronphonon coupling crucially depends on the property of interest. For the $t-J$ model of a high- $T_{c}$ cuprate with a fraction $\delta$ carriers, sum-rules for the imaginary parts of the electron and phonon self-energies show a reduction by a factor $\delta$ for the phonon but not the electron case. This suggests that the apparent $\lambda$ deduced from phonon widths and softenings is reduced by such a factor, while there is no reduction in the electron self-energy. This provides support for phonons being essential for kinks seen in photoemission. Similar arguments suggest that the phonon induced interaction between the carriers is not reduced by a factor of $\delta$, which may be of importance for superconductivity. 
We thank O. K. Andersen, P. Horsch, B. Keimer, N. Nagaosa, D. J. Scalapino, Z.-X. Shen and R. Zeyher for many useful discussions, and Z.-X. Shen for his hospitality during a visit to Stanford.

[1] A. Lanzara, P. V. Bogdanov, X. J. Zhou, S. A. Kellar, D. L. Feng, E. D. Lu, T. Yoshida, H. Eisaki, A. Fujimori, K. Kishio, J.-I. Shimoyama, T. Noda, S. Uchida, Z. Hussain, and Z.-X. Shen, Nature 412, 510 (2001).

[2] S. L. Chaplot, W. Reichardt, L. Pintschovius, and N. Pyka, Phys. Rev. B 52, 7230 (1995); L. Pintschovius, N. Pyka, W. Reichardt, A. Y. Rumiantsev, N. L. Mitrofanov, A.S. Ivanov, G. Collin, and P. Bourges, Physica (Amsterdam) C185-C189, 156 (1991).

[3] L. Pintschovius and M. Braden, Phys. Rev. B 60, R15039 (1999).

[4] L. Pintschovius and W. Reichardt, in Neutron Scattering in Layered Copper-Oxide Superconductors, A. Furrer, Ed., Physics and Chemistry of Materials with Low Dimensional Structures, Vol. 20 (Kluwer Academic, Dordrecht, 1998), p. 165.

[5] R. J. McQueeney, Y. Petrov, T. Egami, M. Yethiraj, G. Shirane, and Y. Endoh, Phys. Rev. Lett. 82, 628 (1999).

[6] Using Eq. (8), a calculated density of states [19] and measured values of the (half-)breathing phonon width [3] and softening one obtains the apparent coupling constant for the phonon self-energy $\lambda^{\Pi} \sim 0.1-0.3$ for $\delta=0.15$. While the apparent coupling for the electron self-energy $\lambda^{\Sigma} \sim 1$ was originally estimated from PES, later work assigned a substantial part of this coupling to lower-lying phonons [20], bringing $\lambda^{\Sigma}$ and $\lambda^{\Pi}$ for the (half-)breathing phonon into better agreement. The observed kink in the PES spectrum can, however, substantially underestimate $\lambda^{\Sigma}$ in Eq. (9). Let the electron self-energy $\Sigma(\omega)=-\left(\lambda^{e l}+\lambda^{\Sigma}\right) \omega$ for small $|\omega|$ and $\Sigma(\omega)=-\lambda^{e l} \omega$ for large $|\omega|$, where $\lambda^{\Sigma}$ is due to the phonon of interest and $\lambda^{e l}$ is due to other effects. Then the observed change of slope at the kink is determined by $1+\lambda^{\Sigma} /\left(1+\lambda^{e l}\right)<1+\lambda^{\Sigma}$, where $\left(1+\lambda^{\Sigma}+\lambda^{e l}\right)=Z^{-1}$, the inverse quasiparticle weight, can be a large number. This suggests that $\lambda^{\Sigma}$ is substantially larger than $\lambda^{\Pi}$.

[7] D. Reznik, B. Keimer, F. Dogan, and I. A. Aksay, Phys. Rev. Lett. 75, 2396 (1995).

[8] T. Cuk, F. Baumberger, D. H. Lu, N. Ingle, X. J. Zhou, H. Eisaki, N. Kaneko, Z. Hussain, T. P. Devereaux, N. Nagaosa, and Z.-X. Shen, cond-mat/0403521

[9] J. E. Han and O. Gunnarsson, Phys. Rev. B 61, 8628 (2000).

[10] F. C. Zhang and T. M. Rice, Phys. Rev. B 37, 3759 (1988).

[11] K. J. von Szczepanski and K.W. Becker, Z. Phys. B 89, 327 (1992).

[12] G. Khaliullin and P. Horsch, Phys. Rev. B 54, R9600 (1996).

[13] P. Horsch, G. Khaliullin, and V. Oudovenko, Physica C 341, 117 (2000).

[14] Z.-X. Shen, A. Lanzara, S. Ishihara, and N. Nagaosa, Philos. Mag. B 82, 1349 (2002).

[15] O. Rösch and O. Gunnarsson, Phys. Rev. Lett. 92, 146403 (2004).

[16] S. Ishihara and N. Nagaosa, Phys. Rev. B 69, 144520 (2004).

[17] We expect that keeping $g_{\mathbf{q}}^{2} / \omega_{p h}$ fixed when changing $\omega_{p h}$ would lead to an unchanged $\lambda$. We have multiplied the $g_{\mathbf{q}}$ rescaled in this way by an additional factor of $\sqrt{2}$. Deducing $\lambda$ from the phonon width then gives a value comparable to what is obtained from experiment.

[18] P. B. Allen, Phys. Rev. B 6, 2577 (1972); Solid State Commun. 14, 937 (1974).

[19] L. F. Mattheiss, Phys. Rev. Lett. 58, 1028 (1987).

[20] X. J. Zhou, J. Shi, T. Yoshida, T. Cuk, W. L. Yang, V. Brouet, J. Nakamura, N. Mannella, S. Komiya, Y. Ando, F. Zhou, W. X. Ti, J. W. Xiong, Z. X. Zhao, T. Sasagawa, T. Kakeshita, H. Esaki, S. Uchida, A. Fujimori, Z. Zhang, E. W. Plummer, R. B. Laughlin, Z. Hussain, and Z.-X. Shen, cond-mat/0405130 